\title{
AUTHOR INDEX Volume 16
}

Agrawal, P. R., see Dhrithi, M. A.

$16(2013) 1330002$

Agrawal, P. R. and Girish, N., Lower Quadrant Dysfunctions Among Dentists Prevalence and Risk Factors

16 (2013) 1350021

Ahmad, K., see Amanullah, Md. F.

16 (2013) 1372001

Ahmed, N., Tufel, S., Khan, M. H. and Khan, P. B., Effectiveness of Neural Mobilization in the Management of Sciatica

16 (2013) 1350012

Aju, K., see A. Dhrithi, M.

Akita, S., see Ota, S.

Aly, T. and Fuji, G., Hip Morphology. Comparative Study between Egyptians and Japanese Adults

16 (2013) 1330002

16 (2013) 1350018

16 (2013) 1350013

Amanullah, Md. F., Ansari, S. and Ahmad, K., Brodie's Abscess Mimicking as of Malignancy: Case Report with Radiological Features

16 (2013) 1372001

16 (2013) 1372001

16 (2013) 1350011

16 (2013) 1350009

Azarian, A., see Saghafi, M.

Baruah, S., Eapen, C. and Kamath, K. R., An Association Between Postural Stability and Sacroiliac Joint Pain - A Case Control Study

Bashford, M. T., see Carroll, M. B.

Belema, T., see Carroll, M. B.

Bello, A. I. and Sepenu, A. S., Mismatch in Body-Chair Dimensions and the Associated Musculoskeletal Pain Among Selected Undergraduate Students in Ghana

Bensamoun, S. F., Dao, T. T., Charleux, F. and Ho Ba Tho, M.-C., Estimation of Muscle Force Derived from In Vivo MR Elastography Tests: A Preliminary Study

Bensamoun, S. F., see Debernard, L.

Caplan, N., see Urwin, S. G.

Carroll, M. B., DeCastro-Pretelt, M., Hust, N., Smith, F., Belema, T., Schramko, M. L., Harris, C. C. and Bashford, M. T., The Relationship of Two Xanthine Oxidase Single Nucleotide Polymorphisms to Hyperuricemia, Gout, and Dose of Xanthine Oxidase Inhibitor

Charleux, F., see Debernard, L.

Charleux, F., see Bensamoun, S. F.

16 (2013) 1350017

16 (2013) 1350004

16 (2013) 1350004

16 (2013) 1350016

16 (2013) 1350015

16 (2013) 1350008

16 (2013) 1350005

Chloros, G. D., see Tanaka, M. L.

Dallas, A. P., see Joseph, O. C.

16 (2013) 1350004

16 (2013) 1350008

16 (2013) 1350015

16 (2013) 1350001

16 (2013) 1350014

Dao, T. T., see Bensamoun, S. F.

16 (2013) 1350015 
Debernard, L., Leclerc, G. E., Robert, L., Charleux, F. and Bensamoun, S. F., In Vivo Characterization of the Muscle Viscoelasticity in Passive and Active Conditions Using Multifrequency MR Elastography

DeCastro-Pretelt, M., see Carroll, M. B.

Der-Boghossian, A. H., see Haidar, R. K.

Dhrithi, M. A., Agrawal, P. R. and Aju, K., Prevalence of Playing-Related Musculoskeletal Disorder (PRMSD) Among Amateur Young Guitar Players

Eapen, C., see Baruah, S.

Equebal, A., see Nezamuddin, Md.

Fuji, G., see Aly, T.

Fujimiya, M., see Suzuki, D.

Gibson, A. St. C., see Urwin, S. G.

Girish, N., see Agrawal, P. R.

Grover, D., see Kachanathu, S. J.

Haidar, R. K., Itani, M., Jabbour, M., Der-Boghossian, A. H. and Khoury, N. J., Review of Diagnostic Approaches for Proliferative Myositis, in the Light of Two Cases

Harris, C. C., see Carroll, M. B.

Hase, K., see Ota, S.

Ho Ba Tho, M.-C., see F. Bensamoun, S.

Homayouni, K., Zeynali, L. and Mianehsaz, E., Comparison Between Kinesio Taping and Physiotherapy in the Treatment of de Quervain's Disease

Hust, N., see Carroll, M. B.

Itani, M., see Haidar, R. K.

Jabbour, M., see Haidar, R. K.

Jariwala, A., see Motwani, P.

Joseph, O. C., Uryasev, O., McNamara, J. P. and Dallas, A. P., Tibial Nerve Perineural Injections at the Posterior Tarsal Tunnel

Kachanathu, S. J., Miglani, S., Grover, D. and Zakaria, A. R, Forearm Band versus Elbow Taping: As a Management of Lateral Epicondylitis

Kader, D. F., see Urwin, S. G.

Kamath, K. R., see Baruah, S.

Kamiya, T., see Suzuki, D.

Khan, M. H., see Ahmed, N.

Khan, M. H., see Nuhmani, S.

Khan, P. B., see Ahmed, N.

Khan, S. A., see Nezamuddin, Md.

Khoury, N. J., see Haidar, R. K.

Kumar, A., see Kumar, S.

Kumar, N. and Mehtani, A., Irreparable Rotator Cuff Tear; A Surgical Dilemma

Kumar, R., see Kumar, S.

Kumar, S., Kumar, A. and Kumar, R., Proprioceptive Training as an Adjunct in Osteoarthritis of Knee

Leclerc, G. E., see Debernard, L.

Massy-Westropp, N. and Toubia, C., Hypermobility as Measured by the Beighton Hypermobility Test is not Predictive of Hand Grip Strength in Young Adults

McNamara, J. P., see Joseph, O. C.

Mehtani, A., see Kumar, N.

Mianehsaz, E., see Homayouni, K.

16 (2013) 1350008

16 (2013) 1350004

16 (2013) 1372002

16 (2013) 1330002

16 (2013) 1350017

16 (2013) 1350011

16 (2013) 1350013

16 (2013) 1350020

16 (2013) 1350005

16 (2013) 1350021

16 (2013) 1350003

16 (2013) 1372002

16 (2013) 1350004

16 (2013) 1350018

16 (2013) 1350015

16 (2013) 1350019

16 (2013) 1350004

16 (2013) 1372002

16 (2013) 1372002

16 (2013) 1350007

16 (2013) 1350014

16 (2013) 1350003

16 (2013) 1350005

16 (2013) 1350017

16 (2013) 1350020

16 (2013) 1350012

16 (2013) 1330003

16 (2013) 1350012

16 (2013) 1350011

16 (2013) 1372002

16 (2013) 1350002

16 (2013) 1330001

16 (2013) 1350002

16 (2013) 1350002

16 (2013) 1350008

16 (2013) 1350006

16 (2013) 1350014

16 (2013) 1330001

16 (2013) 1350019 
Miglani, S., see Kachanathu, S. J.

Mohammadi, G., Musculoskeletal Complaints Among High School Teachers

Motwani, P., Jariwala, A. and Valentine, N., Does Navigation Total Knee Replacement Really Make A Difference?

16 (2013) 1350003

16 (2013) 1350010

16 (2013) 1350007

16 (2013) 1350018

Nezamuddin, Md., Anwer, S., Khan, S. A. and Equebal, A., Efficacy of PressureBiofeedback Guided Deep Cervical Flexor Training on Neck Pain and Muscle Performance in Visual Display Terminal Operators

16 (2013) 1350011

16 (2013) 1330003

16 (2013) 1350022

Nussbaum, M. A., see Toosizadeh, N.

Ota, S., Nakanishi, A., Sato, H., Akita, S., Hase, K. and Suzuki, Y., Differences in Knee Joint Kinematics and Kinetics During Level Walking and Walking with Two Types of Poles - Focus on Knee Varus Moment

16 (2013) 1350018

16 (2013) 1350020

16 (2013) 1350008

Robert, L., see Debernard, L.

Saghafi, M. and Azarian, A., Giant Baker's Cyst and Pseudothrombophlebitis in Patients with Rheumatic Diseases: A New Insight Into Old Complications

16 (2013) 1350009

16 (2013) 1350018

16 (2013) 1350004

16 (2013) 1350016

16 (2013) 1350004

16 (2013) 1350005

Stewart, S., see Urwin, S. G.

Suzuki, D., Watanabe, K., Kamiya, T., Otsubo, H., Yamashita, T. and Fujimiya, M., Angular Stable HTO Plates Differ in Regional Strain According to Geometrical Design

16 (2013) 1350020

16 (2013) 1350018

Tanaka, M. L., Wiesler, E. R. and Chloros, G. D., Biomechanical Consequence of Extensor Pollicis Longus Tendon Relocation

16 (2013) 1350001

Toosizadeh, N. and Nussbaum, M. A., Prolonged Trunk Flexion Can Increase Spine Loads During a Subsequent Lifting Task: An Investigation of the Effects of Trunk Flexion Duration and Angle Using a Sagittally Symmetric, Viscoelastic Spine Model

Toubia, C., see Massy-Westropp, N.

Tufel, S., see Ahmed, N.

Urwin, S. G., Kader, D. F., Caplan, N., Gibson, A. St. C. and Stewart, S., Validation of an Electrogoniometry System as a Measure of Knee Kinematics During Activities of Daily Living

Uryasev, O., see Joseph, O. C.

Valentine, N., see Motwani, P.

Watanabe, K., see Suzuki, D.

Wiesler, E. R., see Tanaka, M. L.

Yamashita, T., see Suzuki, D.

Zakaria, A. R, see Kachanathu, S. J.

Zeynali, L., see Homayouni, K.

16 (2013) 1350022

16 (2013) 1350006

16 (2013) 1350012

16 (2013) 1350005

16 (2013) 1350014

16 (2013) 1350007

16 (2013) 1350020

16 (2013) 1350001

16 (2013) 1350020

16 (2013) 1350003

16 (2013) 1350019 